\title{
Comparison of the effect Of three treatment interventions for the control of Meniere's disease: a randomized control trial
}

\author{
Reham Rafei El Shafei* (i) and Mohamed Qotb
}

\begin{abstract}
Background: Intratympanic (IT) dexamethasone, with or without ventilation tube, is widely used to control vertigo in Meniere's disease (MD) patients. Some authors believe that the tube on its own has a role in the control of MD symptoms. The authors' goal was to compare the effects of them on multiple sets of patients. A three arms randomized control trial was conducted on 60 patients (both males and females; age range, 30-60 years; mean age, 47 years) who were diagnosed with MD according to the 1995 American Academy of Otolaryngology-Head and Neck Surgery (AAO-HNS) Foundation guidelines. The study was conducted at the Otolaryngology Department between May 2016 and May 2018. They were divided into three groups, with 20 patients in each group. The first group received direct IT dexamethasone, the second group received IT dexamethasone through a grommet tube, and the third received saline through grommet.
\end{abstract}

Results: No treatment modality showed statistically significant improvement regarding both hearing and vertigo control at follow-up periods. However, comparison of patients with disease duration of 1 year and patients with duration of 5 years in the total sample of patients showed that patients with 1-year duration showed statistically significant higher percentage of improvement in pure tone audiometry (PTA) and vertigo control at all follow-up periods.

Conclusions: No golden standard in MD treatment; however, initial improvement may be explained as a result of the anti-inflammatory effect of steroids and/or the pressure release due to tube insertion; yet, the progressive course of the disease may diminish that improvement over time. Thus, early intervention either by direct IT injection or through grommet tube or by grommet placement alone can affect treatment outcomes.

Keywords: Meniere's disease, Intratympanic injection, Grommet tube, Vertigo, hearing

\section{Background}

Intratympanic (IT) dexamethasone, with or without ventilation tube, is widely used to control vertigo in Meniere's disease (MD) patients. Some authors believe that the tube on its own has a role in the control of MD symptoms. Our aim is to try to compare the effects of them on multiple sets of patients. Medical treatment of MD controls the disease in approximately two thirds of

* Correspondence: rrs0@fayoum.edu.eg; rehamrafei@yahoo.com ENT Department, Faculty of Medicine, Fayoum University, Fayoum 63514, Egypt patients [1]. A low-sodium diet, avoidance of stress and caffeine, good sleep hygiene, and diuretics are among the most commonly used forms of conservative treatment for $\mathrm{MD}$ in addition to Betahistine tablets in increasing doses. Intraympanic (IT) steroids are a relatively modern form of non-invasive therapy for MD. It is usually saved for resistant cases as a final trial to avoid the more aggressive procedures, like endolymphatic sac surgery [2].

In patients with intractable disease, and if the above measures fail to provide symptomatic control of vertigo,

\section{Springer Open}

(c) The Author(s). 2020 Open Access This article is licensed under a Creative Commons Attribution 4.0 International License, which permits use, sharing, adaptation, distribution and reproduction in any medium or format, as long as you give appropriate credit to the original author(s) and the source, provide a link to the Creative Commons licence, and indicate if changes were made. The images or other third party material in this article are included in the article's Creative Commons licence, unless indicated otherwise in a credit line to the material. If material is not included in the article's Creative Commons licence and your intended use is not permitted by statutory regulation or exceeds the permitted use, you will need to obtain permission directly from the copyright holder. To view a copy of this licence, visit http://creativecommons.org/licenses/by/4.0/. 
ablative therapy with IT gentamicin can be considered. Rarely, vestibular nerve section or labyrinthectomy is required for patients with severe symptoms [3]. IT dexamethasone has minimal risk and is widely used in many centers to control vertigo and has an overall vertigo control success of approximately $80 \%$. IT dexamethasone also has a role in reduction of hearing deterioration [4]. Improvement of vertigo, after ventilation tube placement only without steroid intake in selected patients, has been reported, but the mechanism was controversial $[3,5]$. Moreover, ventilation tubes are useful for delivery of steroid drops to the inner ear [6]. Our review of the literature revealed a number of publications involving the effects of ventilation tube insertion with mixed results $[4,7]$.

Indeed, no evidence-based approach has compared the efficacy of IT dexamethasone injection as opposed to tympanostomy tube insertion in managing patients with MD. We, therefore, studied patients with tympanostomy tube placement and compared our findings with previous reports. The aim of this study was to evaluate the outcomes of different treatment modalities in patients with MD through direct IT dexamethasone instillation or through tympanastomy tube and by tympanostomy tube insertion alone.

\section{Methods}

A randomized control trial was conducted on 60 patients (males and females; age range, 30-60 years; mean age, 47 years) who were diagnosed with $\mathrm{MD}$ according to the 1995 American Academy of Otolaryngology-Head and Neck Surgery (AAO-HNS) Foundation guidelines, as proposed by the Committee on Hearing and Equilibrium.

The study was conducted in the Otolaryngology Department between May 2016 and May 2018. A written consent was obtained from all patients. The study was approved by the local Ethics Committee. Prior to enrollment, all patients had previously received medical treatment for at least 6 months and underwent full audiologic, oto-neurologic, and videonystagmographic (VNG) examinations. Magnetic resonance imaging was done to rule out any retrocochlear disorders.

The patients were randomly divided through computer into three groups; the 20 patients in each group received a different intervention. The first group received IT dexamethasone directly, the second group received IT dexamethasone through a grommet tube, and the third group had a grommet tube placed and received saline. The first group was treated with a fixed protocol of three consecutive weekly IT injections of a $4-\mathrm{mg} / \mathrm{mL}$ dexamethasone preparation as an outpatient. A 25-gauge spinal needle was used for injection with the patient lying supine with the head turned $45^{\circ}$ toward the unaffected ear. Patients were asked not to swallow or move their head to prevent drainage of the solution through the eustachian tube. The patients remained in the supine position for $15 \mathrm{~min}$, after which they were discharged to home.

The second group of patients underwent tympanostomy tube insertion in the affected ear. The tube was placed in the anterio-inferior part of the tympanic membrane under local or general anesthesia. The patients received IT $4 \mathrm{mg} / \mathrm{mL}$ dexamethasone injection through the tympanostomy tube with the same protocol of three consecutive weekly IT injections. The third group underwent tympanostomy tube insertion with $1 \mathrm{ml} \mathrm{sa-}$ line and the same protocol as well.

Treatment outcomes regarding both hearing and vertigo were evaluated according to the AAO-HNS criteria. Hearing evaluation was performed using the AAO-HNS criteria. These criteria considered the following thresholds $(\mathrm{kHz}): 0.5,1 ; 2$, and 4.The patients' hearing levels used four frequencies $(0.5,1,2$, and $4 \mathrm{kHz})$, and pure tone average was calculated before, 3 and 6 weeks and 6 , 12 , and 18 months after treatment. Hearing change was defined as improved (gain of $>10 \mathrm{~dB}$ ), unchanged $( \pm 10$ $\mathrm{dB}$ ), or worse (loss of $>10 \mathrm{~dB}$ ). A numeric value was calculated to measure the effect of treatment on vertigo attacks according to the 1995 American Academy of Otolaryngology-Head and Neck Surgery (AAO-HNS) Foundation guidelines [8]; the average number of definitive attacks per month after treatment was divided by the number of attacks before treatment. The frequency of definitive vertigo attacks was calculated based on the 6 months prior to treatment and 6,12, and 18 months after treatment. Specifically, patients were classified into categories as follows: $0=\mathrm{A}$, complete control; $1-41=\mathrm{B}$, substantial control; 41-80 = C, limited control; 81-120 $=\mathrm{D}$, insignificant control; $>120=\mathrm{E}$, worse (poor) control; and F, secondary treatment initiated because of vertigo-related disability.

\begin{tabular}{llllll}
\hline $\mathbf{0}$ & $\boldsymbol{A}$ & Complete & $\mathbf{8 1 - 1 2 0}$ & $\boldsymbol{D}$ & Insignificant \\
\hline $1-41$ & B & Substantial & $>120$ & E & Poor \\
$41-80$ & C & Limited & & F & Secondary treatment \\
\hline
\end{tabular}

\section{Statistical analysis}

Descriptive statistics were used to describe the characteristics of the study participants and measurements during the study. Mean and standard deviations (SD) are reported for continuous variables. Frequencies with proportions are reported for categorical variables. Median and interquartile range for non-normally distributes continuous variables. Comparison of the numerical variables between the groups is done using one-way ANOVA, while comparison of categorical variables is done using chisquare test and Fisher's exact test. Statistical significance 
was set at $p<0.05$. Analysis was performed using IBM SPSS statistics, version 23 (IBM, Armonk, NY, USA).

\section{Outcomes}

The present study was conducted on 60 patients that were randomly classified through computer into three groups .Table 1 shows comparison of the characteristics of the 3 intervention groups, and there is no statistically significant difference among the groups which indicated proper matching among the 3 groups.

Comparing the improvement in hearing among the 3 groups, Table 2 compared the groups IT Dexa and Dexa through grommet regarding improvement in PTA, and it showed no statistically significant difference among the two groups (Table 3). Moreover, Table 4 compared Dexa through grommet and saline through grommet regarding improvement in PTA and showed also no statistically significant difference between them (Table 5). Tables 6 and 7 compared IT Dexa and saline through grommet regarding improvement in PTA and showed also no statistically significant difference between them.

On the other hand, comparison of the 2 duration groups in the total sample regarding improvement in PTA in Table 8 showed comparison of patient of duration of 1 year and patients with duration of 5 years in the total sample of patients. Patients with 1-year duration showed statistically significant higher percentage of improvement in PTA at all follow-up periods.

Regarding vertigo control, Table 3 compared the groups IT Dexa and Dexa with grommet regarding control of vertigo and showed that no statistically significant difference between them except for the 12 months. At 12 months, all cases of Dexa with grommet showed complete and substantial control; while $30 \%$ of the IT Dexa group showed limited control at this time. On the other hand, Table 5 compared the groups Dexa with the grommet group and grommet with saline regarding control of vertigo and showed that there is no statistically significant difference between them. Table 7 compared the groups IT Dexa group and grommet with saline group regarding control of vertigo and shows that there is no statistically significant difference between them.

However, comparison of the 2 duration groups in the total sample regarding control of vertigo (Table 9) showed comparison of patient of duration of 1 year and patients with duration of 5 years in the total sample of patients. Patients with 1-year duration showed statistically significant better control of vertigo at all follow-up periods.

\section{Discussion}

The poorly understood pathophysiology, as well as the intermittent course of $\mathrm{MD}$, affects our analysis and judgment of all treatment modalities. Hence, an objective conclusion about treatment of MD is often not reached, let alone explained. Over the years, many authors and researchers have studied IT drug transport to the inner ear in the hope of treating or at least controlling MD symptoms. Medical treatment has helped control symptoms, but has had no effect on the long-term evolution of the disease. Invasive procedures, such as labrynthectomy, are controversial, and most clinicians agree that the benefit rarely justifies the potential morbidity.

As regards to measures of PTA, improvement in PTA showed no statistically significant difference among the three groups. On the other hand, comparisons of PTA

Table 1 Characteristics of the study sample: age by ANOVA test, sex and duration by chi-square test, and PTA by Kruskal-Wallis Test

\begin{tabular}{|c|c|c|c|c|}
\hline Characteristics: $n$ (\%) & IT Dexa & Dexa and grommet & saline and grommet & $p$ value \\
\hline \multicolumn{5}{|l|}{ Age } \\
\hline Mean (Sd) & $39.8(8.2)$ & $42.4(7.6)$ & $41.1(7.1)$ & 0.577 \\
\hline \multicolumn{5}{|l|}{ Sex } \\
\hline Male & $10(50 \%)$ & $9(45 \%)$ & $7(35 \%)$ & \multirow[t]{2}{*}{0.622} \\
\hline Female & $10(50 \%)$ & $11(55 \%)$ & $13(65 \%)$ & \\
\hline \multicolumn{5}{|l|}{ Ear } \\
\hline Left & $10(50 \%)$ & $9(45 \%)$ & $8(40 \%)$ & \multirow[t]{2}{*}{0.817} \\
\hline Right & $10(50 \%)$ & $11(55 \%)$ & $12(60 \%)$ & \\
\hline \multicolumn{5}{|l|}{ Duration } \\
\hline 1 year & $10(50 \%)$ & $10(50 \%)$ & $11(55 \%)$ & \multirow[t]{2}{*}{0.935} \\
\hline 5 years & $10(50 \%)$ & $10(50 \%)$ & $9(45 \%)$ & \\
\hline \multicolumn{5}{|l|}{ PTA before } \\
\hline Median (IQR) & $47.5(17.5)$ & $55(13.8)$ & $52.5(10)$ & 0.291 \\
\hline
\end{tabular}

This table shows comparison of the characteristics of the 3 intervention groups, and there is no statistically significant difference between the groups. PTA pure tone audiometrey 
Table 2 Comparing the groups IT Dexa and Dexa through grommet regarding improvement in PTA by chi-square test

\begin{tabular}{llll}
\hline Improved PTA $: n(\%)$ & IT Dexa & Dexa and grommet & $P$ value \\
\hline PTA 3 weeks & $9(45)$ & $10(50)$ & .752 \\
PTA 3 months & $10(50)$ & $10(50)$ & 1 \\
PTA 6 months & $10(50)$ & $10(50)$ & 1 \\
PTA 12 months & $10(50)$ & $10(50)$ & 1 \\
PTA 18 months & $10(50)$ & $10(50)$ & 1 \\
\hline
\end{tabular}

This table shows that there is no statistically significant difference between the IT Dexa group and the Dexa through grommet group regarding improvement in the PTA.

PTA pure tone audiometrey

changes with disease duration (1 year and more than 5 years) in each study group showed statistical significance difference which indicates that patients with shorter disease duration showed relative improvement.

Thus, hearing may improve initially due to the antiinflammatory effect of steroids and/or the pressure release due to tube insertion, but the progressive course of the disease may diminish that improvement over time.

On comparing vertigo control between the three groups with different treatment modalities, there was no statistical significance difference between the study groups as regards follow-up of vertigo after 6, 12, and 18 months after intervention. On the other hand, comparisons of vertigo control with disease duration ( 1 year and more than 5 years) in each study group indicated that patients with shorter disease duration were also improved as there is statistical significance difference in each study group as regards disease duration in all follow-up periods.

Moreover, instillation through ventilation tube or tube insertion alone yielded the same results like direct IT dexamethasone in controlling vertigo attacks. Only disease

Table 3 Comparing the groups IT Dexa and Dexa through grommet regarding control of vertigo by chi-square test

\begin{tabular}{lllll}
\hline & Vertigo & IT Dexa & Dexa and grommet & $P$ value \\
\hline 6 months & Complete & $7(35)$ & $7(35)$ & 0.236 \\
& Substantial & $7(35)$ & $11(55)$ & \\
& Limited & $6(30)$ & $2(10)$ & 0.020 \\
12 months & Complete & $7(35)$ & $7(35)$ & \\
& Substantial & $7(35)$ & $13(65)$ & 1.000 \\
& Limited & $6(30)$ & $0(0)$ & \\
18 months & Complete & $7(35)$ & $7(35)$ & \\
& Substantial & $13(65)$ & $13(65)$ & \\
& Limited & & &
\end{tabular}

This table shows that there is no statistically significant difference between the IT Dexa group and the Dexa through grommet group regarding control of vertigo except for the 12 months. At 12 months, all cases of Dexa through grommet showed complete and substantial control, while $30 \%$ of the IT Dexa group showed limited control at this time.
Table 4 Comparing Dexa through Grommet and saline through grommet regarding improvement in PTA by chi-square test

\begin{tabular}{llll}
\hline $\begin{array}{l}\text { Improved PTA: } \\
n(\%)\end{array}$ & Dexa and grommet & Saline and grommet & $P$ value \\
\hline 3 weeks & $10(50)$ & $13(65)$ & 0.337 \\
3 months & $10(50)$ & $14(70)$ & 0.197 \\
6 months & $10(50)$ & $14(70)$ & 0.197 \\
12 months & $10(50)$ & $14(70)$ & 0.197 \\
18 months & $10(50)$ & $14(70)$ & 0.197 \\
\hline
\end{tabular}

This table shows that there is no statistically significant difference between the Dexa through grommet group and the grommet through the saline group regarding improvement in the PTA.

PTA pure tone audiometrey

duration counted to statistical significant improvement in both hearing and vertigo.

Experimentally induced endolymphatic hydrops in guinea pigs by blockage of endolymphatic ducts was resolved by a tympanostomy tube according to Kimura et al. [8]. Pressure release and/or improved oxygenation of the middle and inner ear is the only justification to the remission that occurred after insertion of the tube. Pressure changes in the middle ear, and the effects on the inner ear require more intense research.

A few authors advocated that intermittent or continuous pressure to the middle ear halts the progression of endolymphatic hydrops [9, 10]. .Russo et al. showed a $26 \%$ resolution of vertigo attacks after insertion of the transtympanic tube, which was a factor in excluding certain patients from a study to evaluate the effect of a low-pressure pulse generator (Meniett). Most authors who have studied the Meniett device have concluded that it is a factor that affects the experimental results [11]. Sugawara et al. offered a similar opinion on ventilation tubes that may improve vertigo, but have no effect on hearing outcomes [12]. In 1998, Thomsen et al.

Table 5 Comparing the groups Dexa through grommet group and saline through grommet regarding control of vertigo by chi-square test

\begin{tabular}{lllll}
\hline & Vertigo & Dexa and grommet & Saline grommet & $P$ value \\
\hline 6 months & complete & $7(35)$ & $7(35)$ & 0.648 \\
& substantial & $11(55)$ & $9(45)$ & \\
12 months & limited & $2(10)$ & $4(20)$ & \\
& complete & $7(35)$ & $7(35)$ & 0.094 \\
& substantial & $13(65)$ & $9(45)$ & \\
& limited & $0(0)$ & $4(20)$ & \\
& complete & $7(35)$ & $7(35)$ & 0.094 \\
& substantial & $13(65)$ & $9(45)$ & \\
\hline
\end{tabular}

This table shows that there is no statistically significant difference between the Dexa through Grommet group and the saline through grommet group regarding control of vertigo. 
Table 6 Comparing IT Dexa and saline through grommet regarding improvement in PTA by chi-square test

\begin{tabular}{llll}
\hline Improved PTA: $n(\%)$ & IT Dexa & Saline and grommet & $P$ value \\
\hline 3 weeks & $9(45)$ & $13(65)$ & 0.204 \\
3 months & $10(50)$ & $14(70)$ & 0.197 \\
6 months & $10(50)$ & $14(70)$ & 0.197 \\
12 months & $10(50)$ & $14(70)$ & 0.197 \\
18 months & $10(50)$ & $14(70)$ & 0.197 \\
\hline
\end{tabular}

This table shows that there is no statistically significant difference between the IT Dexa group and the grommet with saline group regarding improvement in the PTA.

compared the effect of two surgical modalities in 29 intractable MD patients; 15 patients underwent transmastoid endolymphatic sac surgery, while 14 patients underwent tympanostomy tube placement. There were statistically significant reductions in vertigo episodes for patients in both groups postoperatively, but there were no statistical differences between both groups in hearing. The authors concluded that tympanostomy tube placement should be considered as a treatment for MD patients who have vertiginous symptoms refractory to medical treatment [13]. Ogawa et al. advocated that tympanostomy tube treatment, although not effective for all patients, represented a less invasive surgical option with acceptable results [3]. Most studies on this subject, however, do not account for periods of remissions and placebo effects. The general consensus among authors is that ventilation tubes improve vertigo in both humans and animals with endolymphatic hydrops, however, had no effect on hearing results [14-16].

The natural course of the disease is random, and hence, the improvements or lack of improvements cannot be precisely determined by one factor. The lack of objective assessment of the inner ear renders all

Table 7 Comparing the groups IT Dexa group and saline through the grommet group regarding control of vertigo by chi-square test

\begin{tabular}{lllll}
\hline & Vertigo & IT Dexa & Saline grommet & $P$ value \\
\hline 6 months & Complete & $7(35)$ & $7(35)$ & 0.723 \\
& Substantial & $7(35)$ & $9(45)$ & \\
12 months & Limited & $6(30)$ & $4(20)$ & \\
& Complete & $7(35)$ & $7(35)$ & 0.723 \\
& substantial & $7(35)$ & $9(45)$ & \\
& Limited & $6(30)$ & $4(20)$ & 0.094 \\
& Complete & $7(35)$ & $7(35)$ & \\
& substantial & $13(65)$ & $9(45)$ & \\
& Limited & $0(0)$ & $4(20)$ & \\
\hline
\end{tabular}

This table shows that there is no statistically significant difference between the IT Dexa group and the saline with the grommet group regarding control of vertigo.
Table 8 Comparison of the 2 disease duration groups in the total sample regarding improvement in PTA by chi-square test

\begin{tabular}{llll}
\hline Improved PTA: $n(\%)$ & Duration 1 & Duration 5 & $P$ value \\
\hline 3 weeks & $30(97)$ & $2(7)$ & $<0.001$ \\
3 months & $27(87)$ & $7(24)$ & $<0.001$ \\
6 months & $27(87)$ & $7(24)$ & $<0.001$ \\
12 months & $27(87)$ & $7(24)$ & $<0.001$ \\
18 months & $27(87)$ & $7(24)$ & $<0.001$ \\
\hline This table shows comparison of patient with disease duration of 1 year and
\end{tabular}

This table shows comparison of patient with disease duration of 1 year and patients with duration of 5 years in the total sample of patients. Patients with 1-year duration showed statistically significant higher percentage of improvement in PTA at all follow-up periods.

studies subject to a hypothesis that the patients could have been in a period of remission during or at the beginning of the study, and hence, conclusions cannot be accurately drawn from the results.

Although MD is diagnosed by the AAO-HNS clinical criteria and guidelines, ecocochleography could be used to objectively assess inner ear function as demonstrated in multiple animal studies $[3,17,18]$. Multiple research demonstrated the correlation between clinical and electrocochleographic studies in patients with endolymphatic hydrops. The results could represent the baseline for research of treatment modalities irrespective of the fluctuations and periods of remission that occur with all patients with MD; however, the difficulty in recommending guidelines that require EChoG is that this test is not universally available or standardized. The validity of EChoG as serial measure in human subjects with Meniere's disease deserves further study [8].

\section{Conclusion}

No golden standard in MD treatment ; however, initial improvement may be explained as a result of the antiinflammatory effect of steroids and/or the pressure

Table 9 Comparison of the 2 disease duration groups in the total sample regarding control of vertigo by chi-square test

\begin{tabular}{lllll}
\hline & Vertigo & Duration 1 & Duration 5 & P value \\
\hline 6 months & Complete & $17(55)$ & $4(14)$ & 0.001 \\
& Substantial & $12(39)$ & $15(52)$ & \\
\cline { 2 - 3 } 12 months & Limited & $2(7)$ & $10(35)$ & \\
& Complete & $17(55)$ & $4(14)$ & 0.002 \\
& Substantial & $12(39)$ & $17(59)$ & \\
& Limited & $2(7)$ & $8(28)$ & 0.003 \\
& Complete & $17(55)$ & $4(14)$ & \\
& Substantial & $12(39)$ & $23(79)$ & $2(7)$ \\
& Limited & $2(7)$ & &
\end{tabular}

This table shows comparison of patient of disease duration of 1 year and patients with duration of 5 years in the total sample of patients. Patients with 1 -year duration showed statistically significant better control of vertigo at all follow-up periods. 
release due to tube insertion; yet, the progressive course of the disease may diminish that improvement over time. Thus, early intervention either by direct IT injection or through grommet tube or by grommet placement alone can affect treatment outcomes.

\section{Abbreviations}

IT: Intratympanic; MD: Meniere's disease; PTA: Pure tone audiometry; AAOHNS: American Academy of Otolaryngology-Head and Neck Surgery; VNG: Videonystagmographic; SD: Standard deviations

\section{Acknowledgements}

I wish to show my appreciation to Dr Mohamed Elsherif who offered valuable help in the statistics which we used in our work and all our colleagues in the ENT Department, Faculty of medicine, Fayoum University.

\section{Authors' contributions}

All authors contributed to the design and implementation of the research, to the analysis of the results, and to the writing of the manuscript. Dr RS was responsible for the audiological and vestibular assessment. Prof MQ was responsible for the intratympanic injections and tube insertion. Also, the manuscript has been read and approved for submission by all authors.

\section{Funding}

None declared.

\section{Availability of data and materials}

All datasets used are available.

\section{Ethics approval and consent to participate}

The study was approved by the local research ethics board of Fayoum University on February 14th 2016. A written informed consent was obtained from all patients to participate in this work.

\section{Consent for publication}

Not applicable.

\section{Competing interests}

None declared.

Received: 18 March 2020 Accepted: 19 June 2020

Published online: 16 September 2020

\section{References}

1. Rauch SD (2010) Clinical hints and precipitating factors in patients suffering from Ménière's disease. Otolaryngol Clin North Am 43:1011-1017

2. Sharon JD, Trevino C, Schubert MC, Carey JP (2015) Treatment of Menière's disease. Curr Treat Options Neurol 17(4):341

3. Ogawa Y, Otsuka K, Hagiwara A, Inagaki A, Shimizu S, Nagai N, Itani S, Saito Y, Suzuki M (2015) Clinical study of tympanostomy tube placement for patients with intractable Ménière's disease. J Laryngol Otol 129(2):120-125

4. Park JJ, Chen YS, Westhofen M (2009) Meniere's disease and middle ear pressure: vestibular function after transtympanic tube placement. Acta Otolaryngol 129:1408-1413

5. Rutt AL, Hawkshaw MJ, Sataloff RT. Incidence of tympanic membrane perforation after intratympanic steroid treatment through myringotomy tubes. Ear, Nose \& Throat Journal April 2011:90(4):E21

6. Ahsan S, Standring R, Wang Y (2015) Systematic review and meta-analysis of Meniett therapy for Meniere's disease. Laryngoscope. 125(1):203-208

7. Kimura RS, Hutta J (1997) Inhibition of experimentally induced endolymphatic hydrops by middle ear ventilation. Eur Arch Otorhinolaryngol 254(5):213-218

8. Committee on Hearing and Equilibrium. Committee on hearing and equilibrium guidelines for the diagnosis and evaluation of therapy in Ménière's disease. Otolaryngol Head Neck Surg 1995;113:181-185.

9. Casani AP, Piaggi P, Cerchiai N, Seccia V, Franceschini SS, Dallan I (2012) Intratympanic Treatment of intractable unilateral Ménière's disease: gentamicin or dexamethasone? A randomized controlled trial. Otolaryngol Head Neck Surg. 146(3):430-437
10. Casani AP, Cerchiai N, Navari E, Dallan I, Piaggi P (2014) Sellari-Franceschin Intratympanic gentamicin for Meniere's disease: short- and long-term follow-up of two regimens of treatment. Otolaryngol Head Neck Surg. 150(5):847-852

11. Russo FY, Nguyen $Y$, De Seta D, Bouccara D, Sterkers O, Ferrary E, Bernardeschi D. Meniett device in Meniere disease: randomized, doubleblind, placebo-controlled multicenter trial. The Laryngoscope 2017; Feb; 127(2):470-475.

12. Sugawara K, Kitamura K, Ishida T, Sejima T (2003) Insertion of tympanic ventilation tubes as a treating modality for patients with Meniere's disease: a short- and long-term follow-up study in seven cases. Auris Nasus Larynx 30:25-28

13. Thomsen J, Bonding P, Becker B, Stage J, Tos M. The non-specific effect of endolymphatic sac surgery in treatment of Meniere's disease: a prospective, randomized controlled study comparing "classic" endolymphatic sac surgery with the insertion of a ventilating tube in the tympanic membrane. Acta Otolaryngol. 1998;118(6):769-773

14. McRackan TR, Best J, Pearce EC, Bennett ML, Dietrich M, Wanna GB, Haynes DS, Labadie RF (2014) Intratympanic dexamethasone as a symptomatic treatment for Ménière's Disease. Otol Neurotol 35:163

15. Claes GM, De Valck CF, Van de Heyning P, Wuyts FL.The Ménière's Disease Index: an objective correlate of Ménière's disease, based on audiometric and electrocochleographic data. Otol Neurotol. 2011 Jul;32(5):887-92.

16. Kimura $\mathrm{H}$, Aso $\mathrm{S}$, Watanabe $\mathrm{Y}$ (2003) Prediction of progression from atypical to definite Ménière's disease using electrocochleography and glycerol and furosemide tests. Acta Otolaryngol. 123(3):388-395

17. Lopez-Escamez JA, Carey J, Chung W, Goebel JA, Magnusson M, Mandalà M, Newman-Toker DE, Strupp M, Suzuki M, Trabalzini F, Bisdorff A. Diagnostic criteria for Ménière's disease. Journal of Vestibular Research;2015: 25: 1-7 1

18. Kyrodimos E, Aidonis I, Skalimis A, Sismanis A. Use of Glasgow Benefit Inventory (GBI) in Meniere's disease managed with intratympanic dexamethasone perfusion: quality of life assessment. Auris Nasus Larynx; 2011:38:172-17.

\section{Publisher's Note}

Springer Nature remains neutral with regard to jurisdictional claims in published maps and institutional affiliations.

\section{Submit your manuscript to a SpringerOpen ${ }^{\circ}$ journal and benefit from:}

- Convenient online submission

- Rigorous peer review

- Open access: articles freely available online

High visibility within the field

- Retaining the copyright to your article

Submit your next manuscript at $>$ springeropen.com 